Gargano, M. L., Di Gristina, E., Domina, G. \& Venturella, G.: Trees and shrubs in the city of Bari (Italy). — Fl. Medit. 31: 23-30. 2021. — ISSN: 1120-4052 printed, $2240-4538$ online.

Electronic Supplementary File 1.

https://doi.org/10.7320/FlMedit31.023.1

Version of Record published online on 22 March 2021

\title{
The census of trees and shrubs in the city of Bari
}

\begin{tabular}{|c|c|c|c|}
\hline Family & Taxon & Origin & $\begin{array}{l}\text { Biological } \\
\text { Form }\end{array}$ \\
\hline Pinaceae & Abies alba Mill. & Orof. S-Europ. & P scap \\
\hline Fabaceae & Acacia dealbata Link & Australia & P scap \\
\hline Fabaceae & Acacia farnesiana $(\mathrm{L}$.$) Willd.$ & Australia & P caesp \\
\hline Fabaceae & Acacia saligna (Labill.) H.L. Wendl. & Australia & P scap \\
\hline Myrtaceae & Acca sellowiana (O.Berg) Burret & S America & P caesp \\
\hline Sapindaceae & Acer campestre $\mathrm{L}$. & *Europ.-Caucas. & P scap \\
\hline Asparagaceae & Agave americana L. var. americana & Messico & P caesp \\
\hline Asparagaceae & Agave americana var. marginata Trel. & Messico & P caesp \\
\hline Asparagaceae & Agave sisalana Perrine & S America & P caesp \\
\hline Simaroubaceae & Ailanthus altissima (Mill.) Swingle & Asia & P scap \\
\hline Casuarinaceae & Allocasuarina torulosa (Aiton) L.A.S. Johnson & Australia & P scap \\
\hline Asphodelaceae & Aloe arborescens Mill. & S Africa & P succ \\
\hline Asphodelaceae & Aloe vera (L.) Bunn. & Medit. & $\mathrm{P}$ succ \\
\hline Annonaceae & Annona cherimola Mill. & S America & P scap \\
\hline Fabaceae & Anthyllis barba-jovis L. & *W Stenomedit. & P caesp \\
\hline Araucariaceae & Araucaria columnaris (G. Forst.) Hook. & New Caledonia & P scap \\
\hline Araucariaceae & Araucaria heterophylla (Salisb.) Franco & S America & P scap \\
\hline Ericaceae & Arbutus unedo L. & *Stenomedit. & P caesp \\
\hline Sapotaceae & Argania spinosa (L.) Skeels & Africa & P scap \\
\hline Asparagaceae & Asparagus acutifolius L. & *Stenomedit. & G rhiz \\
\hline Asparagaceae & Asparagus aethiopicus L. & S Africa & G rhiz \\
\hline Asparagaceae & Asparagus asparagoides (L.) Druce & Africa & G rhiz \\
\hline
\end{tabular}




\begin{tabular}{|c|c|c|c|}
\hline Poaceae & Bambusa vulgaris Schrad. & Asia & NP \\
\hline Fabaceae & Bauhinia variegata var. candida Voigt & Asia & P scap \\
\hline Berberidaceae & Berberis aquifolium Pursh & N America & NP \\
\hline Berberidaceae & Berberis thunbergii DC. 'Atropurpuea' & Horticultural & NP \\
\hline Berberidaceae & Berberis vulgaris L. & Eursasiat. & NP \\
\hline Nyctaginaceae & Bougainvillea glabra Choisy & S America & P lian \\
\hline Nyctaginaceae & Bougainvillea spectabilis Willd. & S America & P lian \\
\hline Malvaceae & Brachychiton discolor F.Muell. & Tropic. & P scap \\
\hline Malvaceae & Brachychiton diversifolius $\mathrm{R} . \mathrm{Br}$. & Tropic. & P scap \\
\hline Arecaceae & Brahea armata S.Watson & S America & P scap \\
\hline Moraceae & Broussonetia papyrifera (L.) Vent. & Asia & P scap \\
\hline Solanacee & Brugmansia arborea (L.) Steud. & S America & P caesp \\
\hline Arecaceae & Butia capitata (Mart.) Becc. & S America & P caesp \\
\hline Buxaceae & Buxus sempervirens L. & Stenomedit-Subatlant. & $P$ caesp \\
\hline Bignoniaceae & Campsis radicans (L.) Seem. & N America & P lian \\
\hline Betulaceae & Carpinus orientalis Mill. & Pontic & P scap \\
\hline Arecaceae & Caryota urens L. & Asia & P scap \\
\hline Casuarinaceae & Casuarina equisetifolia $\mathrm{L}$. & Asia & P scap \\
\hline Bignoniaceae & Catalpa bignonioides Walter & America & P scap \\
\hline Pinaceae & Cedrus atlantica (Endl.) Carrière & Africa & P scap \\
\hline Pinaceae & Cedrus deodara (D.Don) G.Don & Asia & P scap \\
\hline Pinaceae & Cedrus libani A.Rich. & E-Medit. & P scap \\
\hline Bombacaceae & Ceiba speciosa (A.St.-Hil.) Ravenna & S America & P scap \\
\hline Ulmaceae & Celtis australis $\mathrm{L}$. & *Euri-Medit. & P scap \\
\hline Caesalpiniaceae & Ceratonia siliqua L. & S. Medit. & P scap \\
\hline Fabaceae & Cercis siliquastrum L. & S. Europ. & P scap \\
\hline Cactacee & Cereus repandus (L.) Mill. & S America & P caesp \\
\hline Solanacee & Cestrum parqui L'Her. & S America & NP \\
\hline Arecaceae & Chamaerops humilis L. & W Stenomedit. & P caesp \\
\hline Oleaceae & Chrysojasminum fruticans (L.) Banfi & E Medit. & P caesp \\
\hline Rutaceae & Citrus limon (L.) Osbeck & Asia & P scap \\
\hline
\end{tabular}




\begin{tabular}{|c|c|c|c|}
\hline Rutaceae & Citrus reticulata Blanco & Asia & P scap \\
\hline Rutaceae & Citrus sinensis (L.) Osbeck & Asia & P scap \\
\hline Asparagaceae & Cordyline australis (G. Forst.) Endl. & New Zeland & P caesp \\
\hline Cornaceae & Cornus sanguinea $\mathrm{L}$. & *Eurasiat. & P caesp \\
\hline Poaceae & Cortaderia selloana (Schult. \& Schult.f.) Asch. \& Graebn. & Sudamerica & P caesp \\
\hline Betulaceae & Corylus avellana $\mathrm{L}$. & *Europ.-Caucas. & P caesp \\
\hline Rosaceae & Cotoneaster divaricatus Rehder \& E.H. Wilson & America & $P$ caesp \\
\hline Rosaceae & Crataegus rhipidophylla Gand. & N Europe & P caesp \\
\hline Cupressaceae & Cupressus arizonica Greene & N America & P scap \\
\hline Cupressaceae & Cupressus macrocarpa Hartw. & N America & P scap \\
\hline Cupressaceae & Cupressus sempervirens var. horizonthalis (Mill.) Loudon & Asia & P scap \\
\hline Cupressaceae & Cupressus sempervirens L. var. sempervirens & Euri-Medit & P scap \\
\hline Cupressaceae & Cupressus ×leylandii A.B. Jacks. \& Dallim. & N America & P scap \\
\hline Cycadaceae & Cycas revoluta Thunb. & E Asia & P caesp \\
\hline Cyperaceae & Cyperus papyrus L. & Africa & $\mathrm{He}$ \\
\hline Saxifragaceae & Deutzia scabra Thunb. & E Asia & P caesp \\
\hline Saxifragaceae & Deutzia gracilis Siebold \& Zucc. & E Asia & P caesp \\
\hline Bignoniaceae & Dolichandra unguis-cati (L.) L.G. Lohmann & Messico, S America & P lian \\
\hline Elaeagnaceae & Elaeagnus $\times$ ebbingei J. Door. & Horticultural & P caesp \\
\hline Rosaceae & Eriobotrya japonica (Thunb.) Lindl. & Asia & P scap \\
\hline Myrtaceae & Eucalyptus camaldulensis Dehnh. & Australia & P scap \\
\hline Myrtaceae & Eugenia uniflora $\mathrm{L}$. & Sud America & P caesp \\
\hline Celastraceae & Euonymus japonicus Thunb. & E Asia & P caesp \\
\hline Celastraceae & Euonymus japonicus Thunb. 'Aureo-variegatus' & Horticultural & P caesp \\
\hline Euphorbiaceae & Euphorbia dendroides $\mathrm{L}$. & *Stenomedit.- Macarones. & P caesp \\
\hline Euphorbiaceae & Euphorbia ingens E. Mayer & Africa & $\mathrm{P}$ succ \\
\hline Polygonaceae & Fallopia baldeschuanica (Regel) Holub & Asia & P lian \\
\hline Moraceae & Ficus carica $\mathrm{L}$. & *Medit Turan. & P scap \\
\hline Moraceae & Ficus elastica Hornem. & Asia & P scap \\
\hline Moraceae & Ficus elastica var. decora Guillaumin & Asia & P scap \\
\hline Moraceae & Ficus rubiginosa Desf. & Australia & P scap \\
\hline
\end{tabular}




\begin{tabular}{|c|c|c|c|}
\hline Malvaceae & Firmiana simplex (L.) W.Wight & Asia & P scap \\
\hline Oleaceae & $\begin{array}{l}\text { Fraxinus angustifolia subsp. oxycarpa (Willd.) Franco \& Rocha } \\
\text { Afonso }\end{array}$ & *SE Europ. & P scap \\
\hline Oleaceae & Fraxinus excelsior $\mathrm{L}$. & *Europ.-Caucas. & P scap \\
\hline Oleaceae & Fraxinus ornus L. & *Euri-N-Medit-Pontic & P scap \\
\hline Ginkgoaceae & Ginkgo biloba L. & E- Asia & P scap \\
\hline Fabaceae & Gleditsia triacanthos L. & America & P scap \\
\hline Proteaceae & Grevillea robusta A.Cunn. & Australia & P scap \\
\hline Araliaceae & Hedera helix L. & *Submedit-Subatlant. & P lian \\
\hline Araliaceae & Hedera canariensis Willd. & Canary Is. & P lian \\
\hline Malvaceae & Hibiscus rosa-sinensis L. & E- Asia & P caesp \\
\hline Malvaceae & Hibiscus syriacus $\mathrm{L}$. & Asia & P caesp \\
\hline Aquifoliaceae & Ilex aquifolium $\mathrm{L}$. & *Submedit.-Subatlant. & P caesp \\
\hline Bignoniaceae & Jacaranda mimosifolia D. Don & S America & P scap \\
\hline Asteraceae & Jacobaea maritima (L.) Pelser \& Meijden & *S Medit. & Ch suffr \\
\hline Oleaceae & Jasminum officinale L. & SW Asia. & P caesp \\
\hline Oleaceae & Jasminum polyanthum Franch. & China & P caesp \\
\hline Juglandaceae & Juglans regia $\mathrm{L}$. & SW Asia & P scap \\
\hline Cupressaceae & Juniperus chinensis L. 'Pfitzeriana Glauca' & Horticultural & $\mathrm{NP}$ \\
\hline Cupressaceae & Juniperus horizontalis Moench & America & NP \\
\hline Cupressaceae & Juniperus oxycedrus L. & Euri Medit & P caesp \\
\hline Acanthaceae & Justicia adhatoda L. & Asia & P caesp \\
\hline Sapindaceae & Koelreuteria paniculata Laxm. & Cina, Giappone & P scap \\
\hline Lythraceae & Lagerstroemia indica $\mathrm{L}$. & Asia & P caesp \\
\hline Verbenaceae & Lantana camara $\mathrm{L}$. & Neotrop. & P caesp \\
\hline Verbenaceae & Lantana montevidensis (Spreng.) Brig. & S America & P caesp \\
\hline Lauraceae & Laurus nobilis L. & *Stenomedit. & P caesp \\
\hline Oleaceae & Ligustrum japonicum Thunb. & Asia & P caesp \\
\hline Oleaceae & Ligustrum japonicum Thunb. 'Aureo-variegatum' & Horticultural & P scap \\
\hline Oleaceae & Ligustrum lucidum W.T. Aiton & Asia & P scap \\
\hline Hamamelidaceae & Liquidambar styraciflua $\mathrm{L}$. & America & P scap \\
\hline Arecaceae & Livistona chinensis (Jacq.) Mart. & Asia & P scap \\
\hline
\end{tabular}




\begin{tabular}{|c|c|c|c|}
\hline Hamamelidaceae & Loropetalum chinense f. rubrum H.T. Chang & China & NP? \\
\hline Magnoliaceae & Magnolia grandiflora $\mathrm{L}$ & America & P scap \\
\hline Meliaceae & Melia azederach $\mathrm{L}$. & Asia-Australia & P scap \\
\hline Moraceae & Morus alba L. & Asia & P scap \\
\hline Musaceae & Musa $\times$ paradisiaca $\mathrm{L}$. & Tropics & G rhiz \\
\hline Myrtaceae & Myrtus communis L. & Steno-Medit & $P$ caesp \\
\hline Myrtaceae & Myrtus communis L. 'Tarentina' & *S Italy & $P$ caesp \\
\hline Berberidaceae & Nandina domestica Thunb. & Asia & NP \\
\hline Apocynaceae & Nerium oleander L. & *Stenomedit. & P caesp \\
\hline Oleaceae & Olea europaea $\mathrm{L}$. & *Stenomedit. & P scap \\
\hline Cactacee & Opuntia ficus-indica (L.) Mill. & Neotropic. & P succ \\
\hline Betulaceae & Ostrya carpinifolia Scop. & Pontic & P scap \\
\hline Paeoniaceae & Paeonia $\times$ suffruticosa Andrews & Asia & P caesp \\
\hline Fabaceae & Parkinsonia aculeata $\mathrm{L}$. & Africa/America & P scap \\
\hline Vitaceae & Parthenocissus quinquefolia (L.) Planch. & N America & P lian \\
\hline Vitaceae & Parthenocissus tricuspidata (Siebold \& Zucc.) Planch. & E Asia & P lian \\
\hline Passifloraceae & Passiflora caerulea $\mathrm{L}$. & Sudamerica & P lian \\
\hline Lauraceae & Persea americana Mill. & Sudamerica & P scap \\
\hline Lauraceae & Persea indica (L.) Spreng. & Macaronesia & P scap \\
\hline Hydrangeaceae & Philadelphus grandiflorus Willd. & America & P caesp \\
\hline Lamiaceae & Phlomis purpurea $\mathrm{L}$. & Stenomedit. & NP \\
\hline Lamiaceae & Phlomis viscosa Poir. & Asia & NP \\
\hline Arecaceae & Phoenix canariensis H. Wildpret & Canary Is. & P scap \\
\hline Arecaceae & Phoenix dactylifera $\mathrm{L}$. & Paleo-Subtrop. & P scap \\
\hline Rosaceae & Photinia serratifolia (Desf.) Kalkman 'Red Robin' & China & P scap \\
\hline Poaceae & Phyllostachys aurea Rivière \& C.Rivière & Asia-Australia & NP \\
\hline Poaceae & Phyllostachys nigra (Lind1.) Munro & E Asia & NP \\
\hline Pinaceae & Picea abies (L.) H. Karst. & Eurosib. & P scap \\
\hline Pinaceae & Picea pungens Engelm.'Kosteriana' & Horticultural & P scap \\
\hline Pinaceae & Pinus halepensis Mill. subsp. halepensis & *Stenomedit. & P scap \\
\hline Pinaceae & Pinus halepensis subsp. brutia (Ten.) Holmboe & NE Medit. Mont. & P scap \\
\hline
\end{tabular}




\begin{tabular}{|c|c|c|c|}
\hline Pinaceae & Pinus pinea $\mathrm{L}$. & Eurimedit. & P scap \\
\hline Pinaceae & Pinus canariensis $\mathrm{C} . \mathrm{Sm}$. & Macaronesia & P scap \\
\hline Pinaceae & Pinus nigra J.F. Arnold & E Europ. & P scap \\
\hline Pinaceae & Pinus pinaster Aiton & Stenomedit. & P scap \\
\hline Anacardiaceae & Pistacia lentiscus L. & *Stenomedit. & P caesp \\
\hline Anacardiaceae & Pistacia terebinthus L. & *Eurimedit. & $P$ caesp \\
\hline Pittosporaceae & Pittosporum tobira (Thunb.) W.T. Aiton & E Asia & NP \\
\hline Pittosporaceae & Pittosporum tobira (Thunb.) W.T.Aiton 'Nanum' & Horticultural & NP \\
\hline Platanaceae & Platanus $\times$ hispanica Münchh. & Eurimedit. & P scap \\
\hline Cupressaceae & Platycladus orientalis (L.) Franco & Asia & P caesp \\
\hline Plumbaginaceae & Plumbago auriculata Lam. & S Africa & P lian \\
\hline Polygalaceae & Polygala myrtifolia $\mathrm{L}$. & S Africa & NP \\
\hline Rutaceae & $\begin{array}{l}\text { Poncirus polyandra S.Q. Ding, X.N. Zhang, Z.R. Bao \& M.Q. } \\
\text { Liang }\end{array}$ & Asia & P scap \\
\hline Salicaceae & Populus alba $\mathrm{L}$. & Paleotemp & P scap \\
\hline Salicaceae & Populus nigra L. & Paleotemp & P scap \\
\hline Didiereaceae & Portulacaria afra Jacq. & S Africa & P succ \\
\hline Rosaceae & Prunus dulcis (Mill.) D.A. Webb & S Medit & P scap \\
\hline Rosaceae & Prunus cerasifera Ehrh. & W-Asiat.-Pontic & P caesp \\
\hline Rosaceae & Prunus cerasifera subsp. pissardii (Carrière) Dostál & Horticultural & P scap \\
\hline Rosaceae & Prunus laurocerasus L. & SE Europe, Anatolia & P scap \\
\hline Rosaceae & Prunus mahaleb L. & S. Europ-Pontico & P caesp \\
\hline Rosaceae & Prunus webbii (Spach) Vierh. & E Medit. & P caesp \\
\hline Juglandaceae & Pterocarya fraxinifolia (Poir.) Spach & Asia & P scap \\
\hline Punicaceae & Punica granatum L. & SW Asia & P scap \\
\hline Rosaceae & Pyracantha coccinea M. Roem. & Eurasiat. & P caesp \\
\hline Rosaceae & Pyrus spinosa Forssk. & *Stenomedit. & P caesp \\
\hline Fagaceae & Quercus cerris L. & *N-Eurimedit. & P scap \\
\hline Fagaceae & Quercus ilex $\mathrm{L}$. & *Stenomedit. & P scap \\
\hline Fagaceae & $\begin{array}{l}\text { Quercus ithaburensis subsp. macrolepis (Kotschy) Hedge \& } \\
\text { Yalt. }\end{array}$ & E Medit. & P scap \\
\hline Fagaceae & Quercus pubescens Willd. & *SE Europ. & P scap \\
\hline
\end{tabular}




\begin{tabular}{|c|c|c|c|}
\hline Fagaceae & Quercus trojana Webb & *NE Medit. & P scap \\
\hline Rhamnaceae & Rhamnus alaternus $\mathrm{L}$. & *Stenomedit. & P caesp \\
\hline Fabaceae & Robinia pseudoacacia L. & N America & P scap \\
\hline Rosaceae & Rosa canina $\mathrm{L}$. & *Paleotemp. & NP \\
\hline Rosaceae & Rosa $\times$ hybrida Vill. 'La Sevillana' & Horticultural & NP \\
\hline Asparagaceae & Ruscus aculeatus L. & *Eurimedit. & G rhiz \\
\hline Arecaceae & Sabal palmetto (Walter) Lodd. ex Schult. \& Schult.f. & S America & P scap \\
\hline Salicaceae & Salix babylonica L. & Subtrop-Asia & P scap \\
\hline Lamiaceae & Salvia officinalis L. & *E Stenomedit. & Ch suffr \\
\hline Lamiaceae & Salvia fruticosa Mill. & Macaronesia & P caesp \\
\hline Lamiaceae & Salvia rosmarinus Spenn. & *Stenomedit. & $\mathrm{NP}$ \\
\hline Anacardiaceae & Schinus molle L. & C-S America & P scap \\
\hline Araliaceae & Schefflera arborea (L.) M. Gómez 'Variegata' & Horticultural & P scap \\
\hline Asteraceae & Senecio angulatus L. f. & S Africa & Ch frut \\
\hline Asteraceae & Senecio grandiflorus Meyen & S Africa & Ch frut \\
\hline Cupressaceae & Sequoia sempervirens (D. Don) Endl. & America & P scap \\
\hline Fabaceae & Spartium junceum L. & Eurimedit. & P caesp \\
\hline Strelitziaceae & Strelitzia nicolai Regel \& Körn. & S Africa & P caesp \\
\hline Strelitziaceae & Strelitzia reginae Banks & S Africa & P caesp \\
\hline Fabaceae & Styphnolobium japonicum (L.) Schott & E Asia & P scap \\
\hline Arecaceae & Syagrus romanzoffiana (Cham.) Glassman & S America & P scap \\
\hline Tamaricaceae & Tamarix arborea (Ehrenb.) Bunge & N Africa & P scap \\
\hline Bignoniaceae & Tecoma capensis Lindl. & S Africa & P caesp \\
\hline Lamiaceae & Teucrium fruticans $\mathrm{L}$. & *W Stenemedit. & \\
\hline Cupressaceae & Thuja occidentalis L. & Australia & P caesp \\
\hline Tiliaceae & Tilia americana $\mathrm{L}$. & N America & P scap \\
\hline Tiliaceae & Tilia cordata Mill. & Europ.-Caucas. & P scap \\
\hline Tiliaceae & Tilia platyphyllos Scop. & Europ.-Caucas. & P scap \\
\hline Tiliaceae & Tilia $\times$ europaea L. & Europ.-Caucas. & P scap \\
\hline Apocynaceae & Trachelospermum jasminoides Lem. & China & P caesp \\
\hline Arecaceae & Trachycarpus fortunei Wendt & E Asia & P scap \\
\hline
\end{tabular}




\begin{tabular}{|l|l|l|l|} 
Cannabaceae & Trema micrantha (L.) Blume & C-S America & P scap \\
\hline Ulmaceae & Ulmus canescens Melville & *E-Medit. & P scap \\
\hline Ulmaceae & Ulmus minor Mill. & Europ.-Caucas. & P scap \\
\hline Fabaceae & Vachellia karroo (Hayne) Banfi \& Galasso & S Africa & P caesp \\
\hline Viburnaceae & Viburnum lucidum Mill. & Himalaya & P caesp \\
\hline Viburnaceae & Viburnum rhytidophyllum Hemsl. & Asia & P caesp \\
\hline Viburnaceae & Viburnum tinus L. & *Stenomedit. & P caesp \\
\hline Vitaceae & Vitex agnus-castus L. & *Stenomedit. - Turan. & P caesp \\
\hline Arecaceae & Washingtonia filifera (André) de Bary & P scap \\
\hline Arecaceae & Washingtonia robusta H. Wendl. & N America & P scap \\
\hline Fabaceae & Wisteria sinensis (Sims) Sweet & California & P lian \\
\hline Asparagaceae & Yucca aloifolia L. & Asia & P caesp \\
\hline Asparagaceae & Yucca gigantea Lem. & C America & P caesp \\
\hline Asparagaceae & Yucca gloriosa L. & C America & P caesp \\
\hline
\end{tabular}

* indicates the taxa native to Apulia. 